\title{
Dielectric Properties of Lyotropic Magnetic Liquid Crystal
}

\author{
P. KOPČAnskÝ ${ }^{a}$, L. TOMČO ${ }^{a, b, *}$, J. JADZYN $^{c}$, J. SWIERGIEL ${ }^{c}$, J. MAJOROŠOVÁ ${ }^{a}$,

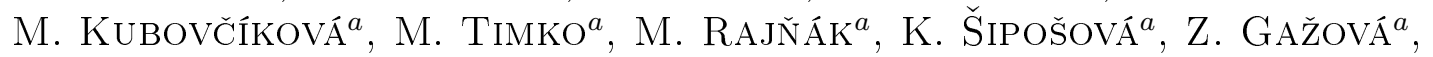 \\ Z. Bednariková ${ }^{a, d}$, N. Tomašovicová ${ }^{a}$, Chin-Kun Hu ${ }^{e}$, S. Hayryan ${ }^{e}$, I. Studenyak ${ }^{f}$, \\ T.M. KOVALCHUK ${ }^{g}$ AND O.V. KOVALCHUK ${ }^{h}$ \\ ${ }^{a}$ Institute of Experimental Physics, Slovak Academy of Sciences, Košice, Slovakia \\ ${ }^{b}$ Faculty of Aeronautics, Technical University, Košice, Slovakia \\ ${ }^{c}$ Institute of Molecular Physics, Polish Academy of Sciences, Poznań, Poland \\ ${ }^{d}$ Department of Biochemistry, Institute of Chemistry, P.J. Safarik University in Košice, Slovakia \\ ${ }^{e}$ Institute of Physics, Academia Sinica, 128 Sec.2, Academia Rd., Nankang, Taipei 11529, Taiwan \\ ${ }^{f}$ Physics Faculty, Uzhhorod National University, Uzhhorod, 88000, Ukraine \\ ${ }^{g}$ Institute of Semiconductor Physics, National Academy of Science of Ukraine, Kyiv, Ukraine \\ ${ }^{h}$ Kyiv National University of Technologies and Design, Kyiv, Ukraine \\ An important feature of lyotropic liquid crystals is the self-assembly of the amphiphilic molecules as su- \\ permolecular structures. We have studied the formation of nematic liquid crystal phase in solutions containing \\ lysozyme amyloid fibrils and magnetic nanoparticles using oscilloscopic method. Interaction of fibrils with mag- \\ netic nanoparticles under the external magnetic field resulted in fibril re-arrangement. The analysis of the obtained \\ results suggests that the decrease in conductivity of solutions in presence of magnetic field is due to decrease of \\ the ion mobility caused by re-arrangement of structures in the solution. The obtained results allow determination \\ of the optimum ratio of the components which can lead to preparation of solutions with a more ordered structure \\ in presence of magnetic field.
}

DOI: 10.12693 /APhysPolA.127.632

PACS: 61.30.Gd, 61.30.St, 75.50.Mm

\section{Introduction}

Experimental studies have shown that the parameters of the liquid crystals (LC) can change significantly under the influence of electric and magnetic fields. The electrooptical effects of various types are at the basis of functioning of most LC devices [1]. Many devices usually include electrodes, which causes a number of problems. Applying magnetic field instead of using electrodes may help to solve these problems because magnetic field penetrates materials with higher efficiency. LC devices sensitive to magnetic field can be used, for example, as baggage scanners in airports, railway stations and other institutions.

The permeability of LC is much smaller than the anisotropy of permittivity. Therefore, the rearrangement of the LC molecules requires large values of the magnetic induction (units, tens of $\mathrm{T}$ ). However, it has been shown that the magneto-optical effect in ferronematics occurs at lower magnetic fields [2]. For preservation of the optical characteristics of $\mathrm{LC}$, the magnetic dopants should not affect the molecular orientation. For this, the dopants must be of small size, and the interaction between them must be weak. Gubin in his review paper showed in detail that the magnetization (per atom) and magnetic anisotropy in magnetic nanoparticles $(\mathrm{MN})$ can be considerably larger than those in the bulk samples [3].

${ }^{*}$ corresponding author; e-mail: ladislav.tomco@tuke.sk
In our study the lyotropic LC (LLC) was used as a matrix. The advantages of these systems are: (i) simple production of systems based on LLC, and (ii) the short switching times under the external magnetic field due to the lower viscosity of water (generally used as a solvent) as compared to the viscosity of the majority of nematic LC. However, LLC with low viscosity coefficient may cause aggregation of nanoparticles in sample solutions. This aggregation of nanoparticles can be avoided by coating nanoparticles with a compound which is poorly wetted by the matrix. It was shown that modifying the surface of magnetic nanoparticles by proteins can prevent their aggregation [4]. Protein does not have necessarily to be coated with magnetic nanoparticles.

The aim of this study was to investigate the influence of magnetic field on the dielectric properties of lyotropic magnetic liquid crystals (LMLC), based on the aqueous solutions of lysozyme fibrils. We also tried to find out if addition of magnetic liquid (ML containing MN dispersed in carrier fluid) can enhance the effect of external magnetic field. We chose the name "lyotropic magnetic liquid crystal" because the ordering of magnetic particles coated with protein in such a system, which is typical for LC, occurs only under the influence of the magnetic field and can be kept for a certain time period after the magnetic field is turned off.

\section{Materials and methods}

In our study we used 6 types of LMLC that differ in content of lysozyme fibrils and magnetic nanoparticles. Three LMLC samples contain only aqueous solutions of lysozyme fibrils at three different concentrations: $0.3,0.7$ 
and $1.4 \mathrm{mM}$. The other three samples were prepared by adding of magnetic liquid $(\mathrm{pH}=2)$ to the first three lysozyme fibrils solutions whereby the weight concentration of the particles in the samples was $0.02 \mathrm{wt} \%$. The magnetic liquid was synthesized by dispersing precipitated $\mathrm{Fe}_{3} \mathrm{O}_{4}$ nanoparticles ( $5 \mathrm{~nm}$ in diameter) in water. In order to avoid any aggregation, the particles were electrostatically stabilized with $\mathrm{HClO}_{4}$. The prepared magnetic liquid was used to enhance the effect of external magnetic field. The studied samples have been labeled as follows: the first 3 characters correspond to the content of lysozyme fibrils, while the last symbol indicates the presence $(\mathrm{M})$ or absence $(0)$ of the magnetic liquid. For example, 07LM corresponds to LMLC with the lysozyme fibrils concentration of $0.7 \mathrm{mM}$, doped with magnetic liquid. For our experiment, we have prepared a special "sandwich" type cell with the distance between the electrodes $3 \mathrm{~mm}$ and with electrode area $0.5 \mathrm{~cm}^{2}$. Filling and washing the cell was done using a syringe. As electrodes we used the ITO layers deposited on glass plates. A few guarding electrodes were also formed on glass plates by etching for elimination of possible edge effects. The guarding electrodes were grounded during the measurements of dielectric properties.

The magnetic field was produced using permanent magnets. Measurements were carried out at the single value of magnetic field induction $0.2 \mathrm{~T}$. The dielectric properties of the obtained samples were examined within the frequency range $10^{-1}-10^{6} \mathrm{~Hz}$ by using oscilloscopic method [5]. The amplitude of the measuring signal of triangular shape was $0.25 \mathrm{~V}$. The measurements were performed at a temperature of $293 \mathrm{~K}$. For each frequency we measured values of the real $(\varepsilon \prime)$ and imaginary $\left(\varepsilon^{\prime \prime}\right)$ components of the complex permittivity.

\section{Results and discussion}

Figure 1 shows the frequency dependence of the components of the complex permittivity $\varepsilon^{\prime}$ (curves 1,3 ) and $\varepsilon^{\prime \prime}$ (curves 2, 4) for distilled (not deionized) water (curves 1,2 ) and lyotropic magnetic liquid crystal 03L0 (curves 3,4 ). The high value of $\varepsilon$ at low frequencies is probably caused by impurities in the water, leading so to the electrode polarization [6]. The $\varepsilon$ / value in this section

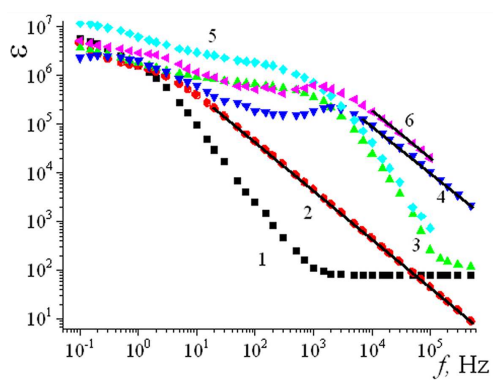

Fig. 1. Frequency dependences of the complex permittivity $\varepsilon^{\prime}$ (curves $1,3,5$ ) and $\varepsilon^{\prime \prime}$ (curves 2, 4, 6) for distilled water (curves 1, 2), 03L0 (curves 3,4 ) and $03 \mathrm{LM}$ (curves 5,6). Regions of the dielectric spectrum where the sample conductivity is determined are marked by the lines. corresponds to the permittivity of liquid bulk. For the sample 03L0, the width of such a section is substantially smaller.

For the frequency dependences of $\varepsilon^{\prime \prime}$, as shown in Fig. 1 (curves 2, 4 and 6), the presence of section of linear dependence on the reciprocal frequency is characteristic (for this frequency range the resistance is independent of frequency). It is in such sections of the dielectric spectrum, the conductivity of the samples was determined.

Comparison of the dielectric spectra of distilled water and that of the sample 03L0 and 03ML shows a relaxation process appearing in the spectra (around $3 \mathrm{kHz}$ ) which can be related to the lysozyme molecules polarization. Moreover, 03LM sample exhibits higher permittivity values in low frequencies. This increase can be associated with a contribution from the polarization of electric double layers formed on the embedded particles. The difference in the dielectric spectra of the 03L0 and 03LM samples allows observing how the LMLC dielectric spectra are sensitive to the added magnetic liquid. However, the low frequency dielectric spectrum of the investigated samples is mainly determined by the solvent (water).

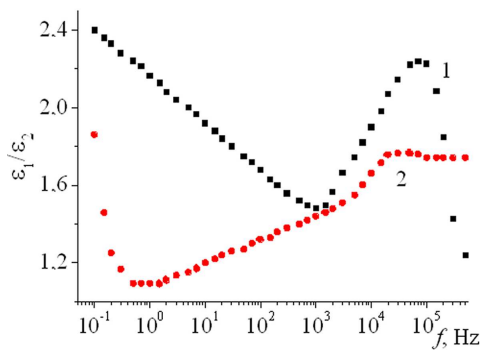

Fig. 2. The frequency dependences of the ratio 14L0 $\left(\varepsilon_{1}\right) / 03 \mathrm{~L} 0\left(\varepsilon_{2}\right)$ for the components $\varepsilon /(1)$ and $\varepsilon^{\prime \prime}(2)$.

Figure 2 shows the ratio of the permittivities of the 14L0 sample $\left(\varepsilon_{1}\right)$ to those of the 03L0 sample $\left(\varepsilon_{2}\right)$ for the components $\varepsilon /(1)$ and $\varepsilon^{\prime \prime}(2)$. From the analysis of these ratios we can conclude that increase in the lysozyme concentration has not lead to simple increase in $\varepsilon l$ and $\varepsilon^{\prime \prime}$ by a certain number of times but to different changes of permittivities in dependence of the frequency. As it was shown in [6], the low-frequency section of the dielectric spectrum corresponds to the processes in the nearelectrode area of the samples, and high-frequency section - to the processes in the bulk of the samples. When the lysozyme fibrils concentration increases, one portion of protein molecules can be adsorbed on the electrodes, which leads to change of the parameters in the nearelectrode area of the samples, and other portion dissolves, which leads to change of the parameters of the bulk of the sample. Adsorption is taken to mean the lysozyme fibrils concentration increases in the near-electrode area of the sample, and not just they deposited on the electrodes. Depending on the lysozyme fibrils concentration in solution, the ratio between the adsorbed and dissolved molecules of dopant is different. This determines the frequency dependence of the ratio of the permittivities for the $14 \mathrm{~L} 0$ and $03 \mathrm{~L} 0$ samples, which is a rather complex and widely different from the monotonous dependence. 


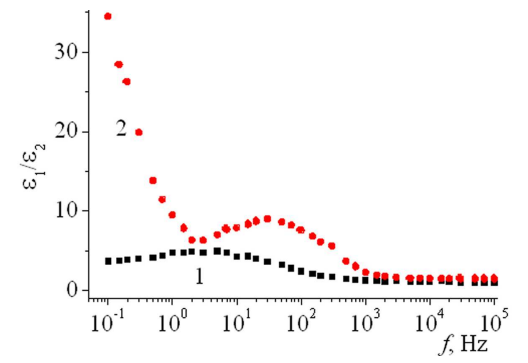

Fig. 3. The frequency dependences of the ratio 14LM $\left(\varepsilon_{1}\right) / 03 \mathrm{LM} \quad\left(\varepsilon_{2}\right)$ for the components $\varepsilon^{\prime}(1)$ and $\varepsilon^{\prime \prime}(2)$.

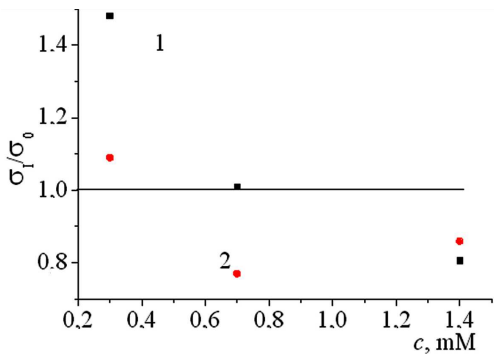

Fig. 4. Dependence of the conductivities ratio in presence $\left(\sigma_{\mathrm{I}}\right)$ and in absence $\left(\sigma_{0}\right)$ of magnetic field on the lysozyme concentration for the samples without (1) and with (2) ML.

Figure 3 shows the ratio of permittivities of the samples 14LM $\left(\varepsilon_{1}\right)$ and 03LM $\left(\varepsilon_{2}\right)$ for the components $\varepsilon /(1)$ and $\varepsilon^{\prime \prime}(2)$. From Figs. 2 and 3 we can conclude that presence of ML can lead to strong redistribution of the amount of molecules adsorbed and dissolved in the bulk. The adsorption of the molecules on the particle surfaces can play an important role in the redistribution effect. As a result, the dielectric spectrum for the ratios of permittivities shown in Fig. 3 is completely different from the spectrum shown in Fig. 2.

Figure 4 shows the dependence of the conductivities ratio in presence $\left(\sigma_{\mathrm{I}}\right)$ and absence $\left(\sigma_{0}\right)$ of magnetic field applied to the lysozyme concentration for samples without (1) and with (2) magnetic liquid (ML). These results indicate the indirect dependence between the decrease in sample conductivity and ordering in LMLC. Lower values of conductivity measured under magnetic field mean a higher level of the order in LMLC samples. The present study of the dielectric properties of LMLC allows us to find the optimum value of lysozyme concentration, which leads to formation of a lyotropic magnetic liquid crystal with a maximally ordered structure at a given concentration of ML.

\section{Conclusions}

We have shown that the presence of lysozyme fibrils and $\mathrm{ML}$ in a magnetic lyotropic liquid crystal changes the frequency dependence of components of the complex permittivity $\varepsilon l$ and $\varepsilon^{\prime \prime}$ for each type of the component. Therefore, one can evaluate the type and concentration of dopants (lysozyme or ML) by using the spectral dependences of the ratio of permittivities of the samples with and without dopants.
It was shown that the constant magnetic field leads basically to the decrease in components of the complex permittivity $\varepsilon$ and $\varepsilon^{\prime \prime}$ within the whole frequency range. In this case the frequency dependences of the permittivities of the samples in presence and absence of magnetic field strongly depend on the presence of ML. We observed that in absence of ML, the frequency dependences are monotonic while in the presence of ML (in the case of $\varepsilon$ and $\left.\varepsilon^{\prime \prime}\right)$ these dependences acquire non-monotonic nature. Moreover, the influence of magnetic field on the samples with ML increases with increasing frequency in the low-frequency part of the dielectric spectrum. This dependence has not been observed for samples without ML. We suggest that the redistribution of the components of lyotropic magnetic liquid crystal between the bulk and near-electrode areas of the sample also greatly affects the change in dielectric spectra in presence of magnetic field.

It is shown that the most univocal dependence on the lysozyme fibrils concentration is the conductivity ratio of the samples in presence and absence of magnetic field $\sigma_{\mathrm{I}} / \sigma_{0}$. For samples without ML, this ratio decreases with increasing lysozyme fibrils concentration. The same concentration dependence for the samples with ML shows a minimum at lysozyme concentration $0.7 \mathrm{mM}$. It is suggested that a decrease in the conductivity of a lyotropic magnetic liquid crystal with increasing lysozyme fibrils concentration is caused by the ordering that depends not only on the protein concentration, but also on the ML concentration. Therefore, the optimal conditions for creation of the most ordered lyotropic magnetic liquid crystal structure are presence of the ML and lysozyme fibrils at concentration $0.7 \mathrm{mM}$.

\section{Acknowledgments}

This work was supported by projects VEGA 0045, 0181, the Slovak Research and Development Agency under the contract No. APVV-0171-10, Ministry of Education Agency for Structural Funds of EU in frame of projects 6220120021, 6220120033 and 26110230097, and M-era.Net project MACOSYS, UPJS-98/13-14. C.K.H. and S.H. are supported by Grant MOST 102-2120-M001-009.

\section{References}

[1] L.M. Blinov, V.G. Chigrinov, Electrooptic Effects in Liquid Crystal Materials, Springer-Verlag, New York 1994.

[2] P. Kopčanský, M. Koneracká, V. Zavisova, J. Jadzyn, G. Czechowski, B. Zywucki, J. Phys. IV (Paris) 7, C565 (1997).

[3] Magnetic Nanoparticles, Ed. S.P. Gubin, Wiley-VCH Verlag, Weinheim 2009.

[4] M. Koneracká, A. Antošová, V. Závišová, G. Lancz, Z. Gažová, K. Šipošová, A. Jurıková, K. Csach, J. Kováč, N. Tomašovičová, M. Fabián, P. Kopčanský, Acta Phys. Pol. A 118, 983 (2010).

[5] A.J. Twarowski, A.C. Albrecht, J. Chem. Phys. 20, 2255 (1979).

[6] A.V. Koval'chuk, J. Phys.: Condens. Matter 13, 10333 (2001). 